EGU21-2852, updated on 31 Mar 2021

https://doi.org/10.5194/egusphere-egu21-2852

EGU General Assembly 2021

(c) Author(s) 2021. This work is distributed under

the Creative Commons Attribution 4.0 License.

\title{
The influence of depth-varying elastic properties in controlling the dynamic rupture of megathrust earthquakes and upper-plate coseismic deformation
}

Manel Prada ${ }^{1}$, Percy Galvez ${ }^{2}$, Carlos Sanchez-Linares ${ }^{3}$, Jean-Paul Ampuero ${ }^{4}$, Valentí Sallarès ${ }^{1}$, Jorge Macias $^{3}$, and Daniel Peter ${ }^{2}$

${ }^{1}$ Barcelona Center for Subsurface Imaging, Institut de Ciències del Mar (ICM), Barcelona, Spain

${ }^{2}$ King Abdullah University of Science and Technology, Physical Science and Engineering Division, Saudi Arabia

${ }^{3}$ EDANYA, Universidad de Malaga, Spain

${ }^{4}$ Université Côte d'Azur and Institut de Recherche pour le Développement, Nice, France

It has been recently proposed that the depth-varying rupture properties of megathrust earthquakes can be explained by the depth distribution of elastic properties of the rocks overlying the megathrust fault. Here we demonstrate that such relationship is mechanically viable by using 3D dynamic rupture simulations. We compare results from two subduction zone scenarios with different depth-distribution of elastic properties to explore the influence of realistic upper-plate elasticity on rupture characteristics such as slip, rupture duration, and frequency content.

The first scenario has a homogeneous distribution of elastic properties, with values of $\mathrm{Vp}, \mathrm{Vs}$, and density typical of rocks overlying the megathrust fault at $25 \mathrm{~km}$ depth. The second scenario includes the typical depth distribution of elastic properties overlying the megathrust fault inferred from worldwide tomographic models of the upper plate. For both scenarios, we simulate three cases with ruptures confined to the shallow domain (0-5 km depth), transitional domain (5-10 km depth), and regular domain (10-25 km depth), respectively. We assume the same friction properties for both scenarios.

Results show that the realistic distribution of elastic properties accounts for increasing slip and decreasing high frequency content trenchwards, and that slip may be 8 times larger and corner frequency 2 times lower in the shallow domain than in the regular domain. Rupture times along depth shows that the rupture through a realistic elastic model may be 2.5-3 times slower in the shallow domain than in the regular domain. Depth-variations of slip, frequency content, and rupture time quantitatively agree with previous predictions, confirming that depletion of high frequency content and slow rupture are inherent of ruptures propagating through the shallow domain, where elastic properties variations drop more rapidly than in the regular and transitional domains.

Depth-dependent elastic properties also affect the dynamics of slip rate. Peak slip rate values in the heterogeneous model anticorrelate with rigidity variations and are 3-4 times higher than those 
observed in the homogeneous model in the shallow domain. Increasing peak slip-rate difference trenchwards correlates with increasing local ground motion differences between models. We also find important differences on permanent coseismic deformation of the upper plate. We show that coseismic deformation is significantly larger in the shallow domain in the heterogeneous models, where uplift ratios may be up to 2 times larger and along-dip displacement of the seafloor may be $>6$ times larger than displacement values from the homogeneous model. We use the permanent uplift seafloor deformation from both models to model the corresponding tsunamis with TsunamiHySEA software. The results show that, at the coast, the maximum amplitude of the tsunami generated by the heterogeneous model may be up to $25 \%$ larger than that excited by the homogeneous model.

This study demonstrate the relevant role of upper-plate elasticity in controlling not only rupture characteristics, but also coseismic upper plate deformation, and tsunamigenesis. Neglecting the distribution of these properties may result in important underestimation of slip, rupture time, and local ground motion, as well as on seafloor coseismic deformation of the shallow domain, which in turn may lead to underestimations of tsunami size. 